\title{
Research dissertation to published paper - the journey to a successful publication
}

David R Radford, ${ }^{1,2 *}$ Robert JG Seath, ${ }^{1,2}$ Latha S Davda, ${ }^{1}$ Gemma Potts ${ }^{1}$

David R Radford ${ }^{1 *}$ AKC, BDS, PhD, FDS, MRD, SFHEA.

1) Principal Lecturer, University of Portsmouth Dental Academy, Faculty of Science and Health, University of Portsmouth. Portsmouth, PO1 2QG.

2) Honorary tutor Faculty of Dentistry, Oral \& Craniofacial Sciences

King's College London. SE1 9RT.

Email david.radford@kcl.ac.uk

Robert JG Seath ${ }^{1}$ BDS, MSc, MFGDP(UK), FHEA.

1) Clinical Director and Lead for Dental Student Education, University of Portsmouth Dental Academy, Faculty of Science and Health, University of Portsmouth. Portsmouth, PO1 2QG.

2) Senor Clinical Teacher, Faculty of Dentistry, Oral \& Craniofacial Sciences King's College London. London. SE1 9RT.

Latha S Davda ${ }^{1}$ BDS, MDS (OMF), MSc, PhD, FDS, Stat UK, FHEA.

1) Clinical Director, University of Portsmouth Dental Academy, Faculty of Science and Health, University of Portsmouth. Portsmouth, PO1 2QG.

Gemma Potts ${ }^{1}$ BA (Hons), MRes.

1) Outreach Officer, University of Portsmouth Dental Academy, Faculty of Science and Health, University of Portsmouth. Portsmouth, PO1 2QG.

*Corresponding author* David R Radford ${ }^{1 *}$ AKC, BDS, PhD, FDS, MRD, SFHEA.

1) Principal Lecturer University of Portsmouth Dental Academy, Faculty of Science and Health, University of Portsmouth. Portsmouth, PO1 2QG.

2) Honorary tutor Faculty of Dentistry, Oral \& Craniofacial Sciences

King's College London. SE1 9RT.

Email david.radford@kcl.ac.uk 


\section{Abstract}

Background Many dental professionals are now completing higher degrees that involve a research project. However, many of those research projects, although worthwhile, are not written up for publication in a peer reviewed journal.

Aim To encourage and assist novice authors in transferring their project report into a paper to submit for publication.

Discussion The relationship between the authors and contributors is considered and advice is given on how to focus on the specific research question and produce a succinct paper within the target Journal's word limit. Emphasis is placed on choosing the right journal for submission and the need to follow the 'instructions to authors' as well as what happens post submission, acceptance and publication. Further some of the difficulties that the authors have encountered on their publication journeys' are highlighted.

Conclusion Great satisfaction will be derived if the novice researcher makes the most of their opportunity of undertaking a research project and subsequently getting it published in a peer reviewed journal. Publishing gives the author recognition within their professional community, a feeling of personal achievement, can create better career perspectives and allows others to build on the work.

Key points

- Encouragement is given to the novice researcher to complete the research cycle by submitting a paper to a peer reviewed journal.

- The use of the Critical Appraisal Skills Programme (CASP) tool and checklists is highlighted.

- Some of the road blocks that a novice researcher will encounter and how to avoid them in order to get their research published are discussed. 


\section{Introduction}

Having completed a successful research project either at undergraduate or postgraduate level, your next achievement should be to publish your work in a respected peer reviewed journal. Whole textbooks ${ }^{1}$ and information on the internet ${ }^{2}$ have been written on how to write a paper for a scientific journal, however, there is little published on how to convert your successful project report or thesis into a paper that is attractive to an editor of a dental, medical or scientific journal. Work published now more than twenty years ago, indeed undertaken in 1996, showed that editors of both general and more specialist dental journals at that time, valued scientific novelty, however, poor research methodology and poor construction of the paper would lead to rejection. ${ }^{3}$

Everyone who has achieved a degree or higher degree that included a project report or dissertation should feel elated. In terms of UK University credits, normally in dentistry an MSc has a 60 credit and an MRes a 100 credit research project at level 7, with a credit defined as 10 notional hours of learning at master's degree level. ${ }^{4}$ Although one can reflect on whether it really did represent 600 or 1000 hours work, it is generally felt by the majority of masters students and their supervisors that it represents a considerable volume of work, more than those notional hours. You are proud of what you researched and you and your supervisors thought it was worthwhile. Of course people who do "real research" like PhDs and 'post docs' will publish, but yours was just an undergraduate, MSc or MRes project! So are you just going to leave it at that and not let the wider scientific community or the population at large know and understand your findings just because it was a so called "small research project"? Is that really very sensible in terms of either the significant resource that went into it, or that someone else will have to repeat your study to find out what you already have established? Even if it is time dependant i.e. current opinions about the use of routine mentoring in dental practice ${ }^{5}$ or the use of Twitter $^{r}$ in the Dental Health Foundation National Smile Month in $2016,{ }^{6}$ the methods that you used may well establish a base line for further research ${ }^{6}$ or that the research can be repeated, extended or applied to other aspects at a later date. ${ }^{5}$

Cunningham's paper $^{7}$ published in the orthodontic literature in 2004 offered great insight into writing a paper for publication but takes a 'de noveau' approach to the task rather than this article which specifically addresses how to convert your current project report into a publication. Over the last ten years, master's projects and PhDs have increasingly developed an approach that either the whole project is submitted as a single paper or a series of stand-alone papers ${ }^{8}$ or includes a potential manuscript for publication as an appendix. ${ }^{9}$ However, recent communication ${ }^{10}$ with a major post graduate provider of dental education in the UK revealed that out of over 100 master's projects 
completed per year, the vast majority were still the traditional full 10-15,000 word project report, with less than $10 \%$ adopting a publication/paper based approach.

Cunningham (2004)7 stated 'So yes just go for it,' however, it is not easy to get published. Your project involved significant hard work and possible heartache, but further it has already been reviewed by more senior academics and examiners and as stated previously, your work should be worthy of being published. However, a word of warning, that although your research project may have been awarded high marks due to the University's assessment matrix, this might have been due to your critical appraisal of the weakness in your research protocol or the research itself. If that is the case you need an early discussion with your supervisors as to whether the additional work involved will lead to a publication.

\section{Preliminaries -get started now}

Getting started is often the most difficult part and for most, beginning with the easiest parts is best i.e. the methods and results followed by the introduction, discussion, conclusion.

However before you start:-

- Read Cunningham's paper ${ }^{7}$ as it contains important information that will not be repeated in this paper.

- Before writing the first draft, discuss with your supervisors the most important elements of your research project that you wish to publish. Considerable help may be gained by asking others, especially those who have published, to critique and proofread drafts. Be aware that editors do not like salami slicing (i.e. dividing up a single research project into multiple paper $^{3}$ and aim for one impactful publication, certainly for a masters project. If you are writing a series of papers from a PhD it is good practice to prepare a number of summaries of the specific areas of the thesis that will be converted to manuscripts and get agreement from your supervisors.

- Writing up your research paper will involve an element of self-management and organisation. Although you have just finished a prolonged period of study the temptation is to take a break, however, the sooner you start to work on creating a paper the fresher it will be in your mind, therefore more relevant and you can still ride the momentum of completing your degree. As with your original project report, make a realistic timetable that includes high quality time and agree it with your fellow authors. Perhaps recruit a 'critical friend' to cagoule you to keep to that timetable. Using simple project management techniques will allow you keep control and work efficiently. Gantt charts are effective in helping you to plan your time visually and meet your deadlines.

- Discuss with your supervisors, now your fellow authors, the journal that you feel is most appropriate. Part of that decision will be the strength of the research project itself such as numbers of participants recruited, return rates of questionnaires, appropriate specimen preparation and laboratory tests or richness of qualitative data. It is important to establish that the topic of the research project will be attractive to the journal that you submit to. If 
the subject is very specific, a specialist journal such as European Journal of Orthodontics or the Journal of Periodontology may be appropriate. If more general, the subject may be of interest to the wider dental population consider the British Dental Journal or if the research is of the most exceptional quality and importance consider Science or Nature. PLOS One is worth considering as it is a peer-reviewed open access scientific journal, published since 2006 and the criteria for publication is based on the quality of the research rather more than specific impact. PLOS One verifies whether experiments and data analysis were conducted rigorously. ${ }^{11}$

- Having selected an appropriate journal, it is essential to carefully read and follow the guidelines for authors. ${ }^{7}$ Failure to comply with the guidelines may result in rejection or require significant corrections, which can delay acceptance and can lead to a loss of enthusiasm. The instructions for authors are essential documents and very comprehensive e.g. Journal of Dentistry runs to 15 pages. ${ }^{12}$ Whilst they may seem laborious to work through, following the prescribed system will save time in the long run.

- Further you need to establish whether you are going to aim for an Open access format journal and agree how the publication charges will be met.

- So what was your research question, as this often forms a great start in producing a running title for you to focus on. You have to produce a 3,000 to 4,000 word manuscript out of your $10,-15,000$ word project report. For example the Journal of Dental Research has a total word limit of 3,200 words for original research reports. ${ }^{13}$ Write your running title down and keep a focus on it.

As it is a tricky and time consuming task to write a paper, it is important for you to consider at an early stage your fellow authors and their order and who you are going to attribute in the acknowledgements. It may be that you write the paper with one or two of those who were involved with the original work, however, it is good practice to acknowledge those who helped you with the research and not just the writing of the paper. Certainly your supervisors will be included but you may also include someone you worked with such as a laboratory technician or a research assistant who was instrumental in helping you. There may be a more senior member of the profession who can help with the paper construction or can act as a 'critical friend' or 'mentor'. If the research was funded by a grant then the name of the funding body should be included.

A manuscript for a scientific journal is typically composed of a number of sections -

- Title, Abstract, Key words

- Introduction

- Methods

- Results

- Discussion, Conclusions

- Acknowledgments, References.

However at this stage, it is a matter of having an outline and 'getting some words onto paper or computer'. It does not matter if the sentences are incomplete and grammar incorrect. The main goal is to capture the main points and ideas. 


\begin{abstract}
Copy and paste you research project abstract into a new master document to start with. This will give you a great feeling as you have started the task but the abstract will probably change. Abstracts are of great importance, as it will lead others to read your work and the final refinement should be undertaken after the main parts of the paper are written. For similar reasons the title of the paper is important but this title does not need to be the same as your dissertation, it probably will not be. Abstracts in many journals are now structured so again refer to 'The instructions to authors' and modify your abstract to those requirements bearing in mind the journal's abstract word limit. You will find that every word counts. The skill is to produce a clear abstract that provides sufficient detail to demonstrate that the design of the study was appropriate and that the evidence to answer the question is strong. Remember abstracts do not normally contain references.
\end{abstract}

\title{
Introduction
}

Most published research papers are about 3,000 to 3,500 words, the higher word counts tend to pertain to qualitative research (i.e. results section with multiple quotes). The purpose of the Introduction is to stimulate interest for the reader. You will need to cut out any extraneous material and make sure your review of the literature is pertinent and concise. Remember Watson and Crick ${ }^{14}$ managed to describe the molecular structure of DNA in only two sides of a journal!

Referring back to your focused research question and running title, your introduction and literature review needs to allow the reader to understand why the research question was developed and how it will start to fill in the gaps in our knowledge. For publication, it is helpful to consider your paper as 'telling a story'. The strong parts in your story line are the introduction and discussion so the links between these sections must be clear. For your original research project, the review of the literature was a work in itself and probably just cutting out words from the original will not work. In most publications there will be about 800 words for this section. A sound approach is to write this section 'de novaeu' as you know and understand the literature far better than when you started your original project. Naturally, write it with your original text in front of you but you need short statements of facts supported by references with no room for long explanations. You may find it useful to have read through your project report literature review and highlighted the most relevant areas-but don't highlight everything! A parallel word document helps with this process. Further try to keep the references down and chose only the most recent and most relevant ones, as for this exercise you are not trying to convince an examiner that you have found out everything that is known about a subject but rather more, what is the most relevant literature to your tight research question. This is not an easy task so this is where you will need to find some high quality time. It is good practice to use a reference manager so the reference list develops as you write. You can introduce other references as new information may have been published.

\section{Methods}

Writing the methods section is much easier. The methods should describe succinctly what was undertaken to allow the reader to be able to reproduce your study; so therefore copy and paste your entire methods chapter and delete the sections that were put in to show the examiners that you were being very thorough i.e. "the bits that your supervisor said you must put in". Also delete any pilot studies unless they were critical to a newly described methodology. Succinctly describe the 
statistical methods used for quantitative research and/or your approach to the analysis of qualitative data. The methods section should be about 500 words unless very complex. A section on any ethical review that you had to obtain needs to be included as well as details of the ethical review body and the reference number.

\section{Results}

Again this section can just be copied and pasted from your original research project, but you need to carefully consider what to take out. Think carefully about the focused research question and the most relevant results. Insert the Tables and Figures from your project report but these will probably have to be reduced down, cut or re formatted as you need to consider how that information is best communicated to the reader. Also again refer to the journal's guide as they will have specific criteria for tables, diagrams and figures including image resolutions. A deciding factor as to whether you include a figure may be one of practicalities of reproducibility and copyright. Include short succinct legends to explain tables and figures. ${ }^{15}$ It is often a great shame to cut out a figure that you were very proud of and took three days of your partner's time to computer generate the graphics, but it may not be key to your focused research question. Make a copy of it, frame it and put it on the wall but take it out of the paper. This is often an opportune time to involve your fellow authors as they will be experienced in preparing a manuscript and they will be able to see 'the wood from the trees'. Further you may wish to refer back to any statistician who helped with the analysis as they are highly analytical and ruthless. For the results of a qualitative study, the number and length of quotes will have to be reduced as well as the subthemes. This will lead to a reduction of the richness of the data but a more precise and impactful paper. This is again a slightly alien concept. A paper is a different beast to an original dissertation but most editors and referees will not accept an overlong results section. The word count for results is often quite variable and also depends on the number of tables and graphs that you include.

\section{Discussion}

Now for the discussion, this should be considered as the brain of your paper. The research question, which was posed, should be answered at the beginning of your discussion. This is where significant re-writing and much editing has to take place. You have to very carefully correlate the literature you cited in your introduction and how your results either, support, refute, or add to our understanding. Care must be taken to provide a commentary and not a reiteration of the results. This is often done best as a totally separate exercise when you have some quality time or often when you are suddenly gifted time by a 'Did not attend of a particularly tricky root canal treatment that you were not looking forwards to'. To do this put your whole discussion into a separate document and read through and read through again, highlighting what you really want to say. Be radical as you only have about 1000 words for the discussion and conclusion. As large sections will have been deleted re visit the entire manuscript and establish whether the discussion now needs to be reordered to make sense. Rosenfeld et al (2000) ${ }^{15}$ offered helpful advice in the construction of your discussion. In addition you need to critically discuss the limitations of your study as well as offering insight as to whether the results are of clinical relevance even if they were statistically significant. ${ }^{7}$ The 
conclusions will probably be similar to the project report but again ensure the focus is on the specific research question of the paper.

\section{The first draft completed}

The bare bones are done, and it has already taken you significant time. Depending on how overcommitted your fellow authors are to their day job of teaching the new cohort of masters students as well as their undergraduate commitments, you could give it to them to read now, however it is a very rough manuscript. If however you are going to lose a month or so in doing so, spend time yourself refining it but give them a heads up that it will be in their in-box in a couple of weeks' time and could they turn it around 'in seven days'? When refining the manuscript, it is often so much better on a hard copy, again when time is suddenly gifted to you. At this stage it is wise to look at the Critical Appraisal Skills Programme (CASP tools) ${ }^{16}$ to ensure that you have included all the pointers that the checklists include for different research methodologies.

\section{Illustrations}

If you have illustrations use then sparingly unless they are critical to your results for example in scanning or transmission electron microscopy studies. ${ }^{17}$ However, make sure that you still have the high resolution original images, as although your manuscript will be reviewed with embedded images, you will need to provide the journal with high resolution images if your paper is accepted.

\section{References}

Normally most masters students use a reference manager to produce their project report so it is a simple task to import the references in using the Journal's house style. There may be a slight variation on a recognised style so ensure your references are exactly to the journal's house style. Be very careful and check them properly against the original references and also make sure that any internet links are not broken before submission.

\section{What happens next?}

You with your fellow authors, refine and re-refine your manuscript ensuring that it makes sense to the international reader (e.g. any jargon is explained, the NHS -- the National Health Service; UDAs -- Units of Dental Activity). If there are multiple authors, try to send the manuscript to them one at time or you will get confused with the number of track changed versions that come back and waste fellow authors' time as they all make the same corrections. Also as you do not wish to lose momentum try to indicate the time scale you are working to. Once the manuscript is agreed upon by all the authors, one of the authors (DRR) was once advised by an eminent professor to put it in the bottom draw for 3 months and then revisit it, and he was a former scientific adviser to this Journal. However, life has moved on but it is still good to give it a two week rest before you make the final read through and check that everything is right. It is amazing how many errors are still present and it is your responsibility as first author to find and correct them.

\section{Submission}

Look carefully at the electronic submission system (now almost universal) before you commit everything to the ether, as some are often not easy to navigate. Include a covering letter to the 
editor with a brief explanation why this manuscript will be of interest to the journal and their readership. All journals have different refereeing processes and these will often be explained in the instructions for authors. ${ }^{12}$ Having submitted you will get an acknowledgement and be able to track your manuscript's progress through the journal publication website (e.g. The British Dental Journal/Nature manuscript tracking website $)^{18}$ but the next stage can often be the most dis-hearting for new authors.

\section{The referees' reports}

Referees are unpaid, they peer review manuscripts and offer their advice to editors, not through spite, but through a genuine true interest in their discipline. If your paper is rejected you will have to discuss with your fellow authors as to how to progress but if accepted with major or minor corrections take these on board and although initially you are disappointed, it invariably leads to a more succinct and better paper. ${ }^{7,15}$ Remember though, you can challenge a recommended change if you feel that the referee is mistaken.

Once re-submitted with corrections and hopefully accepted although this may take a couple of rounds, the next you see the paper is in the so called 'galley proofs' stage but again for new authors this wait seems as if they have forgotten you! This is when the paper is in the final format for publication and you are required to proofread it and are only allowed to make minor changes. Further there may be requests from a sub-editor for you to clarify further points of grammar. You have to do this quickly with your fellow authors, with often less than a five day turn around and it is important that you meet the deadlines. Last there will be a copyright form for you and your fellow authors to sign.

\section{Conclusion}

In this paper we have presented just one way to convert your research report into a publishable manuscript and there will certainly be a myriad of other ways. However, you now have completed the process and have contributed your research of a 'Just small research project' into something that although will not earn a Nobel prize, will certainly contribute to the evidence base in your particular subject. You will be able to follow the number of citations it receives as well as receive notifications of new publications in your area of interest. Overall however, you can feel satisfied that you have made the most of your opportunity of undertaking a research project and all your hard work. Think of the feeling of satisfaction when your friends notice your name in the journal that you have absent-mindedly left on the coffee table! 


\section{References}

1. Day RA. How to write and publish scientific papers. 1998 Mem. Inst. Oswaldo Cruz. Available online at http://www.scielo.br/scielo.php?script=sci arttext\&pid=S0074-

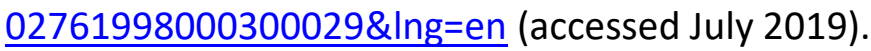

2. Shaikh AA. 7 steps to publishing in a scientific journal. Available online at https://www.elsevier.com/connect/7-steps-to-publishing-in-a-scientific-journal (accessed December 2019).

3. Radford DR, Smillie L Wilson RF, Grace AM. The criteria used by editors of scientific dental journals in the assessment of manuscripts submitted for publication. Br Dent J 1999; 187: 376-379.

4. Academic credit in higher education in England. Available online at https://www.qaa.ac.uk/docs/qaa/quality-code/academic-credit-higher-education-inengland-an-introduction.pdf (accessed July 2019).

5. Seath RJG, Radford DR, Mudford LPA, Reed DP. Should mentoring be routinely introduced into general dental practice to reduce occupational stress? Br Dent J 2019; 227: 121-125.

6. Potts G, Radford DR. \#Teeth\&Tweets:. the reach and reaction of an online social media oral health promotion campaign. Br Dent J 2019; 227: 217-222.

7. Cunningham SJ. How to ... write a paper. J Orth 2004; 31: 47-51.

8. University of Portsmouth. Academic regulations for a doctorate by publications. Available online at

http://www2.port.ac.uk/accesstoinformation/policies/academicregistry/filetodownload,163 744,en.pdf (accessed September 2019).

9. University of Brighton. Academic regulations. Available online at https://staff.brighton.ac.uk/reg/acs/docs/MRes\%20generic\%20framework.pdf (accessed September 2019).

10. Packer ME. Personal communication. King's College London, Faculty of Dentistry, Oral \& Craniofacial Sciences.

11. MacCallum CJ. (2006). ONE for All: The next step for PLOS. PLoS Biol 2006; 4: e401. doi:10.1371/journal.pbio.0040401.PMC1637059.PMID17523266. 
12. Journal of Dentistry Instructions to Authors, Author's information pack. Available online at https://www.elsevier.com/wps/find/journaldescription.cws home/30441?generatepdf=tru e (accessed September 2019).

13. Journal of Dental Research instructions to authors. Available online at https://www.iadr.org/Portals/69/docs/Publications/JDRInstructionstoAuthors.pdf?ver=2017 -02-10-110150-950 (accessed September 2019).

14. Watson JD, Crick FHC. Molecular structure of nucleic acids. Nature 1953; 171: 737-738.

15. Rosenfeldt FL, Dowling JT, Pepe S, Fullerton MJ. How to write a paper for publication. Heart Lung and circulation 2000; 9: 82-87.

16. Critical Appraisal Skills Programme. CASP tools. Available online at https://casp-uk.net/casptools-checklists/ (accessed December 2019).

17. Radford DR, Watson TF, Walter JD, Challacombe SJ. The effects of surface machining on heat cured acrylic resin and two soft denture base materials: a scanning electron microscope and confocal microscope evaluation. J Pros Dent. 1997; 78: 200-208.

DOI:https://doi.org/10.1016/S0022-3913(97)70126-4.

18. The British Dental Journal/Nature manuscript tracking website. Available online at https://mts-bdj.nature.com/cgi-bin/main.plex (accessed January 2020). 